\title{
Advances in Antimicrobial Therapy of Urinary Tract Infection
}

\author{
Ashok M. Karnik, M.D. \\ East Meadow, New York
}

\begin{abstract}
Urinary tract infections (UTI) are one of the most common infections that a clinician is called upon to treat, both in the community and the hospital setting. The prevalence, as well as the spectrum, of etiologic pathogens differs in women and men; for example, E. coli is the causative pathogen in $80 \%$ to $95 \%$ of cases in women, whereas it does so in less than 50\% of cases in men, in whom other Enterobacteriaceae and Gram-positive organisms are more commonly found.

Various studies have improved our understanding of the pathogenesis of UTI which, in turn, has led to a more rational use of the wide variety of antimicrobials now available. The drugs which achieve high intramedullary levels correlate better with the cure in upper tract UTIs. Agents like trimethoprim-sulfamethoxazole and fluoroquinolones eradicate aerobic Gram-negative rods from the fecal and vaginal flora and provide long-term cure. Short-course therapy has now become the standard for most female patients with cystitis, but a 7-14 day therapy is required in other situations.
\end{abstract}

Key words: Urinary tract infection, pathogenesis, therapy, quinolones.

DOI: http://dx.doi.org/10.5915/24-1-15459

Urinary tract infections (UTI) are one of the most common infectious diseases encountered by the clinician today; $25-30 \%$ of women between the ages of 20

From the Department of Medciine

Nassau County Medical Center

East Meadow, NY

Presented at the IMA 24th Annual Convention

Long Island, New York, July 1991

Reprint Requests: Ashok M. Karnik, M.D.

Assistant Professor of Medicine

Department of Medicine

Nassau County Medical Center

2201 Hempstead Turnpike

East Meadow, NY 11554 and 40 give a history suggestive of UTI. ${ }^{\prime}$ These infections account for 5 million office visits yearly in the United States ${ }^{2}$ and evaluation and treatment of ambulatory women with dysuria costs $\$ 1$ billion per year. ' It is a substantial problem in hospitalized patients as well; UTIs are the most common infections occurring in hospitals and nursing homes. In hospitals, about $80 \%$ of nosocomial UTIs are associated with the use of urethral catheters, $5-10 \%$ occur after other genitourinary manipulations and the remainder are not associated with any urological procedures. ${ }^{4}$ In view of their prevalence and cost, ${ }^{5}$ UTIs warrant a review of the current status of their etiology and management.

\section{Definitions}

Urinary Tract Infection refers to the presence of microorganisms in the urinary tract, including the bladder, prostate, collecting system or kidneys. ${ }^{5,6}$ Although most infections are caused by bacteria, 
Table 1. Criteria for defining significant bacteriuria ${ }^{5}$

\section{$\geq 10^{2} \mathrm{CFU}$ coliforms $/ \mathrm{ml}$ or $\geq 10^{5} \mathrm{CFU}$ non-coliforms $/ \mathrm{ml}$ in a symptomatic woman}

\section{$\geq 10^{3} \mathrm{CFU}$ bacteria/ml in a symptomatic man}

$\geq 10^{s} \mathrm{CFU}$ bacteria/ $\mathrm{ml}$ in asymptomatic individuals on two consecutive specimens

Any growth of bacteria on suprapubic catheterization in a symptomatic patient

$\geq 10^{2} \mathrm{CFU}$ bacteria/ml in a catheterized patient.

fungi and viruses may be involved as well. While bacteriuria refers to the presence of bacteria in the urine, the term significant bacteriuria differentiates bacteriuria of true infection from that due to contamination. The probability of infection can be ascertained by quantifying the numbers of bacteria in voided urine. As listed in Table 1, this quantification, defined as colony forming uints per $\mathrm{ml}(\mathrm{CFU} / \mathrm{ml})$, should be interpreted in light of the clinical setting and the manner in which the urine was collected. Asymptomatic bacteriuria refers to significant bacteriuria in a patient without symptoms attributed to the urinary tract. It occurs most commonly in pregnant women and the elderly., ${ }^{7,8}$

UTIs consist of several clinical syndromes. Cystitis is an infection of the urinary bladder, accompanied by symptoms of dysuria, frequency and urgency. However, inflammation of the bladder or urethra in the absence of infection can produce similar symptoms. Also, vaginitis and urethritis caused by pathogens such as herpes simplex, chlamydia trachomatis or Neisseria gonorrhoeae may mimic cystitis. Patients who have symptoms of cystitis, but do not have significant bacteriuria, are described as having acute urethral syndrome. This diagnosis does not exclude cystitis as many such patients are actually found to have bacterial infection of the bladder, although the counts of bacteria in voided urine are lower than the traditional threshold used to define significant bacteriuria $\left(<10^{s} \mathrm{CFU} / \mathrm{ml}\right)$. Acute pyelonephritis is a clinical syndrome which consists of localized flank or back pain associated with systemic symptoms such as fever, chills and prostration. It is caused by infection of the renal parenchyma and collecting system and is often complicated by bacteremia, when it may be called urosepsis. Chronic pyelonephritis, on the other hand, is a pathologic term and refers to the progressive inflammation of the renal interstitium and tubules.

The distinction between uncomplicated and complicated UTIs is important because of its implications regarding the extent of evaluation needed and the choice and duration of treatment. A complicated infection is one that is associated with a condition that increases the risk of developing an infection or hav- ing a persistent infection. Acute cystitis is uncomplicated, but when accompanied by a stone, is considered complicated. Based on localizing studies, it appears that approximately one third of patients who have characteristic symptoms of acute cystitis also have evidence of occult infection of the upper urinary tract. Several factors have been identified that are markers for the presence of occult renal infection or complicated UTI. ${ }^{3}$ (Table 2)

Treatment terminology mainly defines the types of recurrences. Recurrence of bacteriuria with the same organism as originally isolated is termed a relapse, while that with a different one is called reinfection. Chronic UTI describes the situation of a patient with multiple relapses of infection.

\section{Pathogenesis and microbiology of UTI}

The fecal flora serve as a reservoir for uropathogens. These pathogens colonize the vaginal introitus, and, presumably due to the mechanical effect of sexual intercourse, enter the urethra and ascend into the bladder. A host response is stimulated, manifested by symptoms and pyuria, and the patient develops the features of a UTI..$^{9-12}$ Local factors such as vaginal $\mathrm{pH}$ and defense mechanisms of urine and bladder may play an as yet unidentified role in predisposing women to an uncomplicated UTI. ${ }^{13}$

The lower prevalence of UTI in men is attributed to anatomic factors (the greater distance between the anus and the urethral meatus), the drier environment surrounding the male urethra, the greater length of the male urethra, and the antibacterial activity of prostatic fluid. ${ }^{14}$ However, a small number of men in the age range of 20 to 50 years suffer acute uncomplicated UTIs. The two risk factors associated with such infections are homosexuality ${ }^{15}$ and intercourse with an infected female partner. ${ }^{16,17}$

The spectrum of etiologic agents in women is similar in uncomplicated upper and lower UTI' ${ }^{2}$ E. coli is the causative pathogen in approximately $80 \%$ to $95 \%$ and Staphylococcus saprophyticus in $5 \%$ to $10 \%$. Occasionally, other Enterobacteriaciae, such as Proteus mirabilis or Klebsiella sp. or enterococci, are isolated. In $10 \%$ to $15 \%$ of symptomatic patients, no bacterial growth can be detected using routine culture 
Table 2. Factors that suggest the presence of occult renal infection or a complicated urinary tract infection.

Male sex, childhood U.T.I.

Pregnancy

Presentation in an urban emergency department

Hospital-acquired infection

Recent antimicrobial use

Symptoms for more than 7 days at presentation

Indwelling urinary catheter

Recent urinary tract instrumentation

Functional or anatomic abnormality of the urinary tract

Diabetes, immunosuppression

Adapted from Johnson JR, Stamm WE: Diagnosis and treatment of acute urinary tract infections. Infect Dis Clin North Am 1987;1:773-791.

media. E. coli strain isolates from patients with uncomplicated UTI are almost always susceptible to the commonly used oral antimicrobials, although $25 \%$ to $35 \%$ of isolates demonstrate in vitro resistance to ampicillin and sulfonamides. ${ }^{18,19}$ The etiologic agents causing uncomplicated UTIs in men are similar to those in women although the relative prevalence is said to differ. For example, E. coli, although the predominant causative organism, causes infection in less than $50 \%$ of cases, and S. saprophyticus is rarely found. Other Enterobacteriaceae and Gram-positive organisms are more commonly found in men than in women. ${ }^{14}$

\section{Management}

Factors to consider in the selection of antimicrobial agent: When choosing an antimicrobial agent for the treatment of UTI, a number of factors need to be considered. Among these factors are the antimicrobial spectrum of the drug, the duration of adequate urinary levels achieved, the effect of the antimicrobial agent on the fecal and vaginal flora, the potential for side effects and the cost of therapy.

The duration for which the drug concentration in the urine remains above the infecting organism's minimum inhibitory concentration (MIC) is of considerable importance. The drugs which achieve high intramedullary levels are thought to correlate better with cure in upper tract UTI's than those agents which achieve only high serum or urinary levels. Thus, it may be better to choose a drug which achieves high renal tissue levels when treating a patient with a presumptive uncomplicated pyelonephritis. The effect of an antimicrobial agent on fecal and vaginal flora is related to the long-term cure. Agents that have little effect on the anaerobic and microaerophilic normal vaginal flora, but eradicate aerobic Gram-negative rods from the fecal and vaginal reservoir, are most likely to provide longterm cure of uncomplicated UTIs. Trimethoprim- sulfamethoxazole (TMP/SMX) and fluoroquinolones have this property. Both these drugs should not be used in pregnant patients; fluoroquinolones are also contraindicated in patients below 18 years of age. Table 3 compares various antibacterials with regard to the above-mentioned characteristics.'

Routine pre-treatment urine cultures are not necessary in a female patient who presents with pyuria, hematuria or bacteriuria and has a presumptive diagnosis of uncomplicated cystitis. However, culture is necessary in the following situations: in a patient with the presumptive diagnosis of an upper UTI, in a patient with any of the complicating factors listed in the Table 2, and in women whose symptoms do not resolve after a short course of treatment or recur within two weeks. ' Discussed below are various clinical situations and their management.

\section{Acute Cystitis}

In the past, 7-10 day therapy was routinely recommended for lower urinary tract infections. However, in recent years, it has become apparent that most women with lower urinary tract infections have superficial mucosal infection and can be cured by a short course of antibacterial therapy. Single dose therapy achieves high urinary concentrations that are prolonged for at least 12-24 hours and eliminates infection when confined to the bladder. Cure rates range from $60 \%$ to $100 \%$ in women with symptoms of lower urinary infections. ${ }^{20-24}$ Advantages of single dose therapy include lesser expense, better compliance, fewer side effects and perhaps less intense selective pressure for emergence of resistant organisms in the gut, urinary tract and vagina. However, there are possible deleterious effects, such as poorer outcome of infections, that are actually in the upper urinary tract or in the presence of complications like pregnancy, diabetes or an anatomic or functional abnormality of the urinary tract. Two reviews have concluded that three days of therapy may be superior to single-dose therapy. ${ }^{25,26}$ Some of the preferred agents for three days of therapy are TMP/SMX (one double-strength tablet, twice a day), cephalexin (250-500 mg four times a day), amoxicillin-clavulanic acid (250-500 mg amoxicillin/125 mg clavulanic acid three times a day), norfloxacin (400 mg twice a day) or ciprofloxacin ( $500 \mathrm{mg}$ twice a day). It has been recommended that for the single dose (one day) therapy TMP/SMX or trimethoprim alone be used and other agents for the three-day course. ${ }^{27}$

Ampicillin and amoxicillin are not recommended for the three-day course because of the following reasons: there is a high prevalence among community-acquired UTIs of ampicillin-resistant uropathogens, beta-lactams are present in the urine in high concentration for a much shorter period than TMP/SMX and they do not eradicate E. coli from 
Table 3. Characteristics of antimicrobial agents used for treatment of uncomplicated urinary tract infection ${ }^{1}$ (Modified from Hooton TM and Stamm WE, 1991)

\begin{tabular}{|c|c|c|c|c|c|c|}
\hline & $\begin{array}{c}\text { Sulfa- } \\
\text { methoxazole }\end{array}$ & Amoxicillin & $\begin{array}{l}\text { Nitro- } \\
\text { furantoin }\end{array}$ & $\begin{array}{l}\text { Amoxicillin/ } \\
\text { Clavulanate }\end{array}$ & TMX/SMX & $\begin{array}{c}\text { Fluoroquin- } \\
\text { olones }\end{array}$ \\
\hline \multicolumn{7}{|l|}{ Uropathogen } \\
\hline Resistance & $25 \%$ & $30 \%$ & $5 \%-15 \%$ & $5 \%$ & $5 \%-15 \%$ & $2 \%$ \\
\hline \multicolumn{7}{|l|}{ Urine } \\
\hline Concentration* & High & High & High & High & High & High \\
\hline Half-life in urine & +++ & ++ & + & ++ & +++ & +++ \\
\hline \multicolumn{7}{|l|}{$\begin{array}{l}\text { Intestinal/Vaginal } \\
\text { Effect }\end{array}$} \\
\hline Anaerobes & - & ++ & - & +++ & - & - \\
\hline Coliforms & + & + & - & + & +++ & +++ \\
\hline Side Effects & ++ & ++ & ++ & +++ & ++ & ++ \\
\hline Cost** & Low & Low & Low & High & Low & High \\
\hline
\end{tabular}

Key: - = No apparent effect; $+=$ Short half-life or minimal antimicrobial effect; $++=$ Moderate half-life, antimicrobial effect, or side effects; $+++=$ Long half-life, strong antimicrobial effect, or frequent side effects. *All these antimicrobial agents achieve urinary concentrations ranging from $40 \%$ to $95 \%$. $^{31 * *}$ The drug cost for a three day regime is less than $\$ 3.00$ for agents classified as "low" cost and above $\$ 10.00$ for the ones classified as "high" cost."

the vaginal and fecal reservoirs, thereby resulting in higher relapse rates.'

Various studies have suggested that short-course therapy should become the standard for most female patients with suspected lower urinary tract infection. However, short-course therapy has not been adequately evaluated in men and is not recommended at present. Similarly, short-course therapy is not appropriate for women who have a previous history of UTI caused by an antibiotic-resistant organism or who have had symptoms for more than seven days. ${ }^{27}$ In these patients (who have an increased likelihood of upper UTI) and in males, 7-10 days of therapy are recommended. Routine post-treatment cultures are not necessary in a case of cystitis, but if the symptoms do not respond or recur, a urine culture should be obtained. In pregnant women, men, children and patients at high risk for renal damage, a follow-up culture should be routinely obtained 1-2 weeks after discontinuation of therapy to detect relapses.

\section{Acute uncomplicated pyelonephritis}

Some patients with uncomplicated pyelonephritis can be managed as outpatients on oral therapy. For patients who are severely ill and cannot take oral hydration and medication or where there is concern about the diagnosis, compliance or social circumstances, the patient should be hospitalized for parenteral treatment. The traditional regimen of ampicillin and gentamicin provides a broad coverage but has limited usefulness because of the high prevalence of ampicillin resistance in uropathogenic E. coli, the greater expense of administering combinations of an- timicrobials compared with a single agent and the infrequency of enterococci and P. aeruginosa in uncomplicated infections. It has been suggested that, if the Gram stain is not suggestive of infection caused by Gram-positive pathogens, treatment should be initiated in the patient with acute uncomplicated pyelonephritis with TMP/SMX, a third generation cephalosporin or an aminoglycoside. ${ }^{\prime}$ As soon as the patient has improved symptomatically and has become apyrexial, usually at 24 to 48 hours, therapy can be switched from parental to oral. One of the following broad-spectrum oral drugs can be used:TMP/SMX, ciprofloxacin or norfloxacin. The opinions regarding the exact duration of treatment differ, but if a patient shows a dramatic response with resolution of fever and symptoms within a day or two of initiating therapy, treatment may be discontinued after 10 to 14 days. In most nonpregnant adults who remain asymptomatic, follow-up cultures are optional, but they are mandatory within one to two weeks of completion of therapy in pregnant women, children and patients with recurrent symptomatic pyelonephritis in whom maintenance therapy is planned. ${ }^{27}$

\section{Recurrent Uncomplicated Urinary Tract Infection}

If a patient relapses after therapy for a symptomatic UTI, the most likely possibilities are 1) renal involvement, 2) a structural abnormality of the urinary tract, for example calculi, or 3) chronic prostratitis. While some authors ${ }^{27}$ feel that every patient with acute pyelonephritis should have at least an ultrasound examination to rule out obstruction or 
stone, others ${ }^{1}$ have rarely found urologic abnormalities in those who respond promptly to antimicrobial therapy and recommend an evaluation only after two recurrences of pyelonephritis. Studies with excretory urography and cystoscopy in women with recurrent UTI have demonstrated abnormalities in less than $5 \%$ of patients, few of which are correctable. ${ }^{28}$

Patients with a recurrent uncomplicated UTI may be managed by continuous or post-coital prophylaxis or intermittent self-treatment, the selection of the method depending upon the frequency and pattern of recurrences. Women who report an apparent temporal association between infection and intercourse would be more likely to benefit from the post-coital approach. Those who get more than three UTIs per year should receive continuous prophylaxis for about six months, while those with less than three episodes per year, may be advised to treat themselves with a single dose or a three-day regimen at the first symptom of UTI and to call their physician if the symptoms have not completely resolved by 48 hours.

\section{UTI in pregnancy}

All pregnant patients with significant bacteriuria must be treated with an appropriate antimicrobial agent with the aim of maintaining sterile urine throughout gestation. Nontoxic drugs like sulfonamide, ampicillin, cephalexin or nitrofurantoin may be used for a single dose or three days of therapy. Sulfonamides should not be administered in the last few weeks of gestation and tetracycline should be avoided altogether. A urine culture should be obtained one to two weeks after discontinuation of therapy and then monthly for the remainder of gestation. In patients with multiple relapses, radiologic evaluation should be considered postpartum. Catheterization should be avoided at the time of delivery. ${ }^{27}$

\section{Asymptomatic bacteriuria}

The approach to this problem depends upon the age of the patient. While it is recommended that children be treated with a single dose or short-course therapy, it is considered a benign disease in the elderly and need not be treated. In adults, asymptomatic bacteriuria needs treatment in the presence of obstruction or pregnancy. In non-pregnant women, if two urine cultures confirm the presence of bacteriuria, a non-toxic agent should be chosen, but if the micro-organism is resistant to all but the toxic agents, then treatment should not be instituted.

\section{Uncomplicated UTI in adult men}

UTIs are uncommon in adult men. Those that occur in the male newborn, the infant and the elderly male are associated with urologic abnormalities, bladder outlet obstruction or instrumentation.
Management of UTI in men differs in certain aspects from that in women. A urinary gram-stain is indicated to detect the occasional infection caused by enterococci. Any of the previously recommended agents may be used with the exception of nitrofurantoin, which does not achieve reliable tissue levels and hence would not be useful in occult prostratitis. Ampicillin and/or amoxicillin are indicated if enterococci are considered as probable pathogens and a sevenday regimen is recommended because of the greater likelihood of an occult complicating factor. It is also wise to routinely obtain pre- and post-treatment cultures. Recurrence with the same organism suggests a prostatic or upper urinary tract source of infection and warrants a 4 to 6 week regimen of either TMX/SMX or a fluoroquinolone. ${ }^{1}$

\section{Conclusion}

Recent studies have improved our understanding of the pathogenesis of UTI which has had an impact on the management of this common problem. It has become clear that cystitis is a superficial mucosal infection, that pre - and post-treatment cultures are not needed routinely and that short-course therapy is highly effective in this situation. On the other hand, women with upper UTI and the newborn, the infant and the elderly need to be treated for seven to fourteen days. TMP/SMX and fluoroquinolones, which eradicate aerobic Gram-negative rods from the fecal and vaginal flora, produce best long-term cures. Fluoroquinolones, the latest addition to our armamentarium, have a favorable profile (Table 3); there is low incidence of uropathogen resistance, they achieve a high urinary concentration and have a long half-life. For the treatment of uncomplicated UTI, norfloxacin, ciprofloxacin and ofloxacin have been highly effective ( 92 to $100 \%$ ) and comparable to TMP/SMX. In complicated UTIs treatment with fluoroquinolones is considered superior to treatment with TMP/SMX, beta-lactam antibiotics or other drugs in their ability to eliminate bacteriuria. ${ }^{29}$ Ciprofloxacin, the only fluoroquinolone currently available in USA in both IV and oral form $^{30}$, can result in substantial cost-saving when used sequentially (IV/oral) in the treatment of serious infections.

\section{References}

1. Hooton TM, Stam WE: Management of acute uncomplicated urinary tract infection in adults. Med Clin North Am 1991;75:339-57.

2. Cypress BK: Patient's reasons for visiting physicians: National Ambulatory Medical Care Survey. United States, 1977-1978. In: Vital and Health Statistics. Data from the National Health Survey, Series 13(56)(DHHS Publication No. 82-1717). Hyattsville, MD, National Center for Health Statistics, 1981.

3. Johnson JR, Stamm WE: Diagnosis and treat- 
ment of acute urinary tract infections. Infect Dis Clin North Am 1987;1:773-91.

4. Warren JW: Nosocomial urinary tract infections. In Mandell GL, Douglas RG, Bennett JE. Ed. Principles and Practice of Infectious Diseases. 3rd ed. 2205-13. Churchill Livingston, New York 1990.

5. Johnson CC: Definitions, classification and clinical presentation of urinary tract infection. Med Clin North Am 1991;75:241-52.

6. Blowers R, Asscher, AW, Brumfitt W et al.: Recommended terminology of urinary-tract infection. Br Med J 1979;2:717-19.

7. Andriole VT: Urinary tract infections in pregnancy. Urol Clin North Am 1975;2:485-98.

8. Boscia JA, Kobasa WD, Knight RA et al.: Epidemiology of bacteruria in an elderly ambulatory population. Am J Med 1986;80:208-14.

9. Stamey TA, Timothy M, Millar M, et al.: Recurrent urinary tract infections in adult women: the role of introital enterobacteria. Calif Med 1971; 115:1-19.

10. Nicolle LE, Harding GKM, Preiksaitis J et al.: The association of urinary tract infection with sexual intercourse. J Infect Dis 1982;146:579-83.

11. Remis RS, Gurwith MJ, Gurwith D. et al.: Risk factors for urinary tract infection. Am J Epidemiol 1987;126:685-94.

12. Strom BL, Collins M, West SL, et al.: Sexual activity, contraceptive use and other risk factors for symptomatic and asymptomatic bacteruria. A case-control study. Ann Intern Med 1987; 107:816-23.

13. Sobel JD: Pathogenesis of urinary tract infections: Host defenses. Infect Dis Clin North Am 1987;1:751-72.

14. Lipsky BA: Urinary tract infections in men: Epidemiology, pathophysiology, diagnosis and treatment. Ann Intern Med 1989;110:138-50.

15. Barnes RC, Diafuku R, Roddy RE, et al.: Urinary tract infection in sexually active homosexual men. Lancet 1986;1:171-73.

16. Bailey RR, Peddie BA, Swainson CP et al.: Sexual acquisition of urinary tract infection in a man. Nephron 1986;44:217-18.

17. Wong ES, Stamm WE: Sexual acquisition of urinary tract infection in a man. JAMA 1983; 250:3087-88.

18. Fowler JE: Urinary tract infections in women. Urol Clin North Am 1986;13:673-83.
19. Gruneberg RN: Antiobiotic sensitivities of urinary pathogens, 1971-82. J Antimicrob Chemother 1984;14:17-23.

20. Slade N, Crowther ST: Multicenter survey or urinary tract infections in general practice. $\mathrm{Br}$. J Urol 1972;44:105-9.

21. Ronald AR, Boutrous P, Mourtada H.: Bacteriuria localization and reponse to single dose therapy in women. JAMA 1976; 235:1854-56.

22. Fang LST, Tolokoff-Rubin NE, Rubin PH.: Efficacy of single-dose and conventional amoxicillin therapy in urinary-tract infection localized by the antibody-coated bacteria technique. $\mathrm{N}$ Engl J Med 1978;298:413-16.

23. Harbord RB, Gruneberg RN.: Treatment of urinary tract infection with a single dose of amoxicillin, cotrimoxazole or trimethoprim. $\mathrm{Br}$ Med J 1981;283:1301-02.

24. Grossius G.: Single dose nitrofurantoin therapy for urinary tract infections in women. Curr Ther Res. 1984;35:925-31.

25. Philbrick JT, Bracikowski JP.: Single dose antibiotic treatment for uncomplicated urinary infections. Less for less? Arch Intern Med 1985; 145:1672-78.

26. Stamey TA.: Recurrent urinary tract infections in female patients: an overview of management and treatment. Rev Infect Dis 1987;9(Supp 2):S195-S208.

27. Sobel JD, Kaye D: Urinary tract infections. In Mandell GL, Douglas RG, Bennett EJ, ed. Principles and Practice of Infectious Diseases. 3rd Ed p. 582-611. Churchill Livingstone N.Y. 1990.

28. Fowler JE, Pulaski ET: Excretory urography, cystography and cystoscopy in the evaluation of women with urinary tract infection: A prospective study. N Engl J Med 1981;304:462-65.

29. Hooper DC, Wolfson JS: Fluoroquinolone antimicrobial agents. N Engl J Med 1991; 324:384-94.

30. Anonymous: Intravenous ciprofloxacin. The Medical Letter on Drugs and Therapeutics. 1991; 33:75-6.

31. Mandell GL, Sande MA: Antimicrobial agents. In: Gilman AF, Rall TW, Nies AS, Taylor P. Ed Goodman and Gilman's. The pharmacological basis of theraputics. New York, Pergamon Press, 1990:1047-97. 